\title{
Zweite Abtheilung.
}

Botanik und Pharnakognosie.

\section{Notiz über Semen Cinae; \\ ron}

Fried. Bassermann in Manheim.

Von einem Freunde, welcher seit 14 Jahren Reisen in das innere Rufsland macht, erhielt ich folgende Notiz über Semen Cinae, welche ich der Veröffentlichung werth halte: s dafs die Bucharen und Kirgisen ihn aus den Umges bungen von Turkestan ron einer wildwachsenden - Planze, Dschusan (Artemisia), sammeln, die beson» ders gut forthommt in den Sandsteppen Kara und - Kisil, und, obgleich bitter, fast das einzige Futter ^ der Pferde und Kameele dort ausmacht. Die Caravanen * bringen den Semen Cinae auf die Tauschmärkte von - Troitzk, Orenburg, Petropawlowsk und Bemipalatinst. - Nach St. Petersburg kommt er über Nischni Nowgorod.๙ Der Semen Cinae, ron welchem hier die Rede ist, ist der sogenannte Aleppische oder Levantische. Ich wollte schon längst diese Bezeichnung als unpassend răgen, da man ihn gar nicht aus der Levante beziehen kann, sondern immer über Petersburg, und schlage hiemit vor, ihn Semen Cinae moscoviticum zu nennen.

\section{Die deutsche Coloquinte;}

\author{
ron \\ Prof. Dierbach.
}

Die I'nanze, von welcher ich hier einige Notizen mitzutheilen wage, gelöı t zu den ältesten, deren die Geschichte 
des deuischen Feld- und Gartenbaues gedenkt, die ollem. Ansehen nach auch noch immer hie und da cultirirt wird, aber von den Bolaniliern und Aerzten lange vergessen oder übersehen wurde.

Wer nur einigermałsen mit der älteren Geschichte unsers Vaterlandes belannt ist, dem wird es nicht fremd seyn, dafs in den ersten Jalrehunderten der christlichen Zeitrechnnng Künste und Wissenschaften in Deutschland haun bekannt waren, oder doch in sehr rohem 'sustande sich hefanden. Einer der ersten beschützer und Beförderer derselben war Harl der Grofse (geboren $\mathbf{7 4 3}$ za Ingelheim bei Mainz, yestorben am 38. Januar 814), der durch so viele glänzende Eigenscbaften ausgezeichnet, die ihn über seine Zeitgenossen erheben, auch als der wahre Begrïnder und Stifter des deutschen Gartenbaues angesehen werden tann, zu dessen Uoterstützung and Belebung ibn besonders brittische Benedictiner Mönche vermochten, die damals und zwar nicht mit Unvecht für rorzügliche Fienner der Wisseoschaften galten und Harls ganzes Vertrauen besafsen. Von ihnen mag wohl eigentlich jenes berühmte Cupitulare de Villis abstammen, in weichem die Gewächse einzeln aufgezählt werden, die nach dem Willen des Herrschers in dea Gärten der Laiserlichen Pfalzen werden sollten. Hier bammen denn nun auch Coloquinten (coloquintides) ror, die den Auslegern jener alten Urkunde nicht wenig Mühe machten, denn die gewöhnliche Coloquinte (Cucumis Colocynthis L) Hann damit nnmöglich gemeint scyn, indem sie aus den wärmeren Gegenden Asiens und Afrilsa's stammead nimmermebr in Dentschland unter freiem Hirnmel gezogen werden kann, und ron T'reibläusern oder ähnlichen Anstalten zur Cultur der Gewächse wärmerer Himmelsstriche wat damais überall noch heine Rede. Sclbst der berühmte und licuntnilsreiche $s p r e n g e l$ gibe darüber 
keinen Aufschlufs, indem er *) jene Coloquintides ganz burz mit einem Fragezeichen abfertigt, ohne sich auf eine nähere Erörterung einzulassen.

Indessen hat doch schon der alte deutsche Botaniker Hieronymus Tragus, der im 16 ten Jahrhundert lebte, die hier in Rede stehende Pllanze deutlich und genau unter dem Namen diutsche Coloquinte beschrieben; seine Nacbrichton darüber siad so interessant, dafs ich das Wesentlichste davon mittbeilen will. Er beschreibt zurörderst die orientalische Colorguinte and setzt dann hinzu, von dieser sey die deutsche Coloquinte durchaus rerschieden, cowohl in Hinsicht der Blätter und Frucht, als wie überhaupt der ganzen Gestalt nach. In Speier werde sio ron Einigen aus Liebhaberei gezogen; ihr Suame sey breit wie bei den Citrullen; ibre Blätter seyen nicht aschgrau, sondern schwäralich, raub, an Gestaht denen der gelben Cucumern ähnlich. Die Stengel, sagt er ferner, sind rond und ranb, sie briechen amher wie die der Zaan. rübe, nicht leicht winden sie sich an etwas hinauf, breiten sich aber mit ihren Ranken so sehr aus, dafs sie eine weite 8trecke Feides um sich her bedecken. Sie haben zweierlei Blanen, die deneı des Kürbisses ahnlich sehen, eine Form derselben hat bein Knöpfchen (germen) und bringt auch keine Frucht (flor. masculus), aus der andern aber bilden sich die Coloquinten, die eine birnförmige Gestalt haben und so grofs sind wie Regelsbirnen. Wenn sie im Herbste zeitig sind, 80 haben sie eine schön gelbe Farbe, sind innen schwammig, mit vielen Saamen angefüllt und sehr bitter. Die Pllanze wächst gerne an sandigen feuchten Orten, uad gedeiht so gat, dafs Tragas nicht selten ron einem einzigen Stocke 200 solcher Coloquinten erhielt.

*) Geschicbte der Sotanik Bd. I. S. 196. 
Wenn man, bemerkt Tragus ferner, sich der Coloquinten bedienen will, so sind diese deutschen den fremden vorzuriehen, da sie gelinde und gelahrlos sowohl die Galle als den Schleim ausführen. Beim Gebrauche soll man eine reife Coloquintenfrucht aushöhlen, das Mark nebst den Saa. men mit einem Becher Wein übergiefsen, 24 Stunden lang stehen lassen, dann auspressen, dann Abends beim Schlafengehen trialien, worauf am näcbsten Morgen ein gelinder Durchfall erfolze, durch den die Unreinigkeiten, Schloim, Galle u. \&. w., gehörig ausgeführt würden.

Lobelins, der etwas später als Tragus lebte, kennt dieselbe Pflanze and erinnert, dafs sie in Doutschland, Frankreich und England gezogen werde, wo sie im September reife; aber er hält sie koineswegs für eín, der wahren orientalischen Coloquinte vorzuziehendes Purgirmittel; auch kenne er mehrere Varietäten derselben, worunter vielleicht eigone Arten sich hefinden möchten. Dodonaeus bildet unsere deutsche Coloquinte nnter dem Namen Cucurbita silvestris ab, und redet ebeafalls von ihrer Bitterkeit, so wie ron ihren Purgirkräften. In dem Pinax des Caspar Baubin. (8. 3،3) heifat sie Colocynthis pyriformis seu Pepo umarus.

Erwägt man alles das, was über die deutsche Coloquinte von den alten Schriftstellem gesagt wird, so lälst sie sich am füglichsten auf Cucurbita ovifera L. varietas pyriformis Seringe beziehen, deren Mutterpflanse um Astrachan wild wachsen soll; in Frankreich zicht man sie oft, und zwar unter dem Namen Cougourdette; es scheint daron Spielarten zu geben, die keineswegs so bitter sind, wie die alten Botaniker angeben.

Wohl zu unterscheiden von dieser ist übrigens die bleine (Coloquinelle) oder falsche Coloquinte (fausse Coloquinte) der Franzosen (Cucurbita Colocyntha, Risso !l. 377.), die anch 
Orangine heifst, eine bugelige Gestalt hat und in Hinsicht der Grörse und Farbe mit den Citronen oder Pomeranzen übereinkommt, weshalb sie Willdenow Cucurbita aurantia uennte.

\section{Dritte Abtheilung.}

Therapie und Araneiformeln.

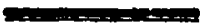 \\ Beobachtungen über das Cyankalium als Arz- neimittel; \\ Félix Boudet.}

Das Cyanbalium ist seit einigen Jahren als ein wabres Specificum gegen Nerralgie betrachtet worden. Sowobl äufserlich wie innerlich angewendet ist die vorgeschriebene Gabe immer sehr klein, and es ist deshalb ron der gröfsten Wichtigkeit, dafs seine Zusammensetzung, von der seine Wirkung abbängt, immer gleich sey, denn die geringsten $\Delta b$ weichungen darin könnten die gefäbrlichsten Folgen für den Kranken baben. Um hiervon eine Idee zu geben, mag es genügen, sich der Beobachtangen zu erinnern, welche Herr Dr. Trou vé an der zten medicinischen Schule zu Caen an Herrn Orfila berichtet hat, und welche in dem Journal de chimie médical (t. X. pag. 23) bekannt gemacht worden sind.

Man kann aus diesom Beispiel den grofsen Unterschied in der Wirkang beurtheilen, wolcher zwischen dem fucuchten, nach der ältern Verfahrungsart dargestelten, und dem trock. 